PROFESIONALES Y HERRAMIENTAS PARA EL DESARROLLO LOCAL Y SUS SINERGIAS TERRITORIALES. EVALUACIÓN Y PROPUESTAS DE FUTURO IX Coloquio Nacional de Desarrollo Local del GTDL-AGE 

ANTONIO MARTÍNEZ PUCHE, XAVIER AMAT MONTESINOS, ISABEL SANCHO CARBONELL y DANIEL SANCHIZ CASTAÑO (EDS.)

\section{PROFESIONALES Y HERRAMIENTAS PARA EL DESARROLLO LOCAL Y SUS SINERGIAS TERRITORIALES. EVALUACIÓN Y PROPUESTAS DE FUTURO}

IX Coloquio Nacional de Desarrollo Local del GTDL-AGE

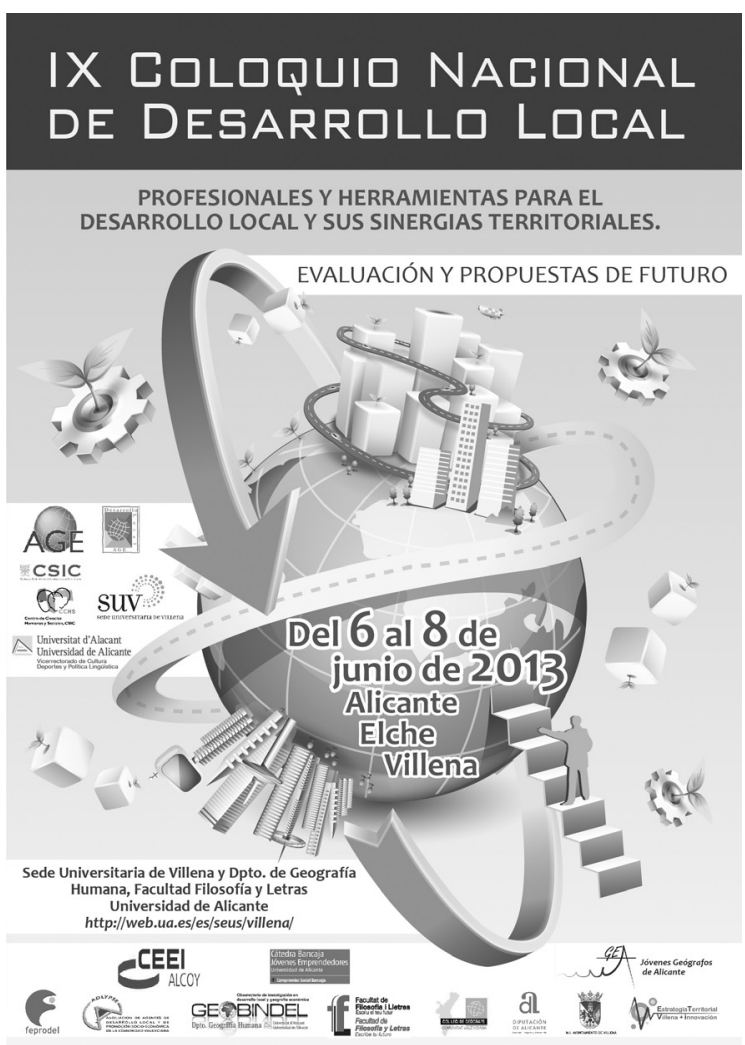


Este libro ha sido examinado y valorado por evaluadores ajenos a la Universidad de Alicante, con el fin de garantizar la calidad científica del mismo.

Publicacions de la Universitat d'Alacant

03690 Sant Vicent del Raspeig

Publicaciones@ua.es

http://publicaciones.ua.es

Telèfon: 965903480

(C) Antonio Martínez Puche, Xavier Amat Montesinos,

Isabel Sancho Carbonell y Daniel Sanchiz Castaño (eds.), 2016

(C) d'aquesta edició: Universitat d'Alacant

ISBN: 978-84-16724-00-0

Dipòsit legal: A 92-2016

Disseny de coberta: candela ink

Composició: Página Maestra (Miguel Ángel Sánchez Hernández)

Impressió i enquadernació: Guada Impresores

\section{unte \\ Unión de Editoriales
Universitarias Españolas \\ WWW.une.es
WWA}

Esta editorial es miembro de la UNE, cosa que garantiza la difusión y comercialización nacional y internacional de sus publicaciones.

Reservados todos los derechos. Cualquier forma de reproducción, distribución, comunicación pública o transformación de esta obra sólo puede ser realizada con la autorización de sus titulares, salvo excepción prevista por la ley. Diríjase a CEDRO (Centro Español de Derechos Repográficos, www.cedro.org) si necesita fotocopias o escanear algún fragmento de esta obra. 


\title{
MODELO DE NEGOCIO AGROALIMENTARIO PARA EL DESARROLLO LOCAL DE LA PROVINCIA DE MANABÍ. ECUADOR
}

\author{
Mg. Sebastiana de Monserratte Ruiz Cedeño \\ Universidad Técnica de Manabí. Ecuador. moncitaruiz@gmail.com \\ Mg. Vicente Félix Veliz Briones. \\ Universidad Técnica de Manabí. Ecuador. vicenteveliz@gmail.com \\ PhD. Norberto Pelegrín Entenza \\ Universidad Técnica de Manabí. Ecuador. norbepelegrin@gamil.com \\ MSc. Yurisley Toledo Leal. \\ Universidad Técnica de Manabí. Ecuador. yurisleytoledo@gmail.com
}

\section{RESUMEN}

La investigación presenta los fundamentos teórico metodológicos que la sustentan a partir de la toma de posición de los autores, Se abordan los modelos de negocios en el sector agroalimentario, se enfatiza, en la importancia modelos asociativos para el contexto ecuatoriano y de la provincia de Manabí que se erijan a partir de la potenciación de la innovación y la gestión del conocimiento para el desarrollo local sostenible ,así como la necesidad de transitar hacia buenas prácticas de gestión y lograr encadenamientos productivos que beneficien a los productores.

Palabras claves: Modelo, Agronegocios, Desarrollo Local, Gestión del conocimiento, Asociatividad. 


\section{AbSTRACT}

The research presents the theoretical and methodological foundations that support from the stance of the authors, the business models in the food industry are addressed, it emphasizes the importance associative models to the Ecuadorian context and in the province of Manabi that is erected from enhancing innovation and knowledge management for sustainable local development, and the need to move towards good management practices and achieve productive chains that benefit producers.

Keys Words: Model, Agribusiness, Local Development, Knowledge Management, associativity

\section{INTRODUCCIÓN}

Para el logro exitoso en el desempeño de una empresa influyen diferentes factores que determinan la competitividad de la misma. Estos factores o elementos conforman el eje direccional que implica la obtención del éxito para cualquier tipo de empresa, independientemente del sector o área donde se desarrolle o pretenda conformar su programa de servicios o ventas. Dentro de estos factores se destacan por su importancia el diseño del producto o servicio que brinda la organización o estructura diseñada para el manejo de sus costos y gastos, el diseño adecuado de un plan financiero que respalde los propósitos de la organización y contribuya al cumplimiento de las metas trazadas, la estructura y el entorno competitivo de la industria, la fortaleza y emprendimiento de su líder así como el diseño de un modelo de negocio que contribuya al desarrollo innovador de la empresa.

En el desarrollo de las empresas y las proyecciones de nuevos negocios se ha considerado comúnmente el desarrollo o elaboración de planes y estrategias a partir del diseño de planes de negocios. Estos son conceptos que han cambiado con los años llegando a tomar mayor protagonismo el diseño de modelos de negocios los cuales son considerados la herramienta innovadora y sostenible generadora de valor para el cliente, la sociedad y los inversionistas.

$\mathrm{El}$ artículo pretende ofrecer reflexiones teóricas sobre modelos asociativos agroalimentarios cuyo centro es la gestión del conocimiento y su importancia para el desarrollo local endógeno de la provincia de Manabí. Ecuador.

\section{Desarrollo}

\subsection{Consideraciones en torno a modelos de negocios}

Siguiendo la definición planteada por el autor Alexander Osterwalder (2010), "un modelo de negocio es aquel que describe la base lógica de cómo una empresa crea, entrega y captura valor. Este modelo debe ser innovador, rentable 
y escalable, con el objetivo de buscar alternativas estratégicas para llevarlo a cabo". (Osterwalder, 2010).

La diferencia entre el plan de negocios y el modelo de negocio ha marcado pautas en el desarrollo empresarial donde se ha ascendido del documento llamado plan de negocio el cual representa aspectos como la descripción y análisis de la industria, la propuesta de productos o servicios, análisis de la competencia, investigaciones de mercado y análisis de posicionamiento, siendo una herramienta útil para identificar las posibles oportunidades de negocio, al modelo de negocio innovador, el cual genera alto valor para la empresa, los clientes y los diferentes grupos de interés.

De acuerdo a lo planteado por Daniela Ruiz Massieu (2011) en su artículo Modelo de Negocio o Bussines Plan el diseño del modelo de negocio fomenta la innovación y, por ende, la creación de valor para la empresa, para los clientes y para sus grupos de interés. La rapidez con la que esos modelos innovadores han transformado algunas industrias no tiene precedente; así, en la actualidad, la definición de un modelo de negocio innovador es crucial: aun cuando el producto o servicio por sí mismo no sea innovador, el modelo y las estrategias puestas en marcha para comercializarlo pueden serlo. Un mismo producto, servicio o tecnología puede tener varios modelos de negocio, por lo que es trascendental desarrollar aquel que logre la mayor creación de valor. De hecho, un gran número de las empresas definidas como las más innovadoras y creativas del mundo no han comercializado productos o servicios innovadores, sino que han logrado su éxito, con lo que han generado un inmenso valor e incluso han revolucionado sus industrias, basados en modelos de negocio innovadores. (Ruiz Massieu, 2011).

En opinión de los autores, los modelos de negocios en la actualidad han venido a transformar la gestión empresarial, considerándose una herramienta para el despegue del desarrollo de las empresas a través de su atributo innovador lo cual ha fortalecido el desempeño de las compañías con la generación de valor para todos los miembros de la cadena de gestión.

Daniela Ruiz Massieu (2011) destaca también la relevancia y aporte que representa para las empresas el uso adecuado del modelo de negocio cuando plantea:

Un punto importante es que el modelo de negocio debe conceptualizarse antes de la elaboración completa del business plan; de hecho, debe ser el primer paso, una vez que se ha identificado la oportunidad de negocio. El modelo de negocio precisará cómo funcionará la empresa, cómo generará dinero y cómo creará valor; es un programa de acción que identifica el proceso de la creación de valor y sirve como eje rector para instrumentar las estrategias a través de estructuras organizacionales, de procesos y de sistemas. (Ruiz Massieu, 2011)

Los autores coinciden con el planteamiento anterior ya que considera que el empleo de modelos de negocios brinda oportunidades a las empresas para 
una mejor planificación y orientación estratégica, lo cual puede potenciar favorables resultados a través de un manejo adecuado de sus sistemas, estructuras y procesos generadores de valor.

El sector agrícola se ha caracterizado a lo largo de los años por su carácter socioeconómico el cual es un pilar importante en el desarrollo de los pueblos, principalmente los subdesarrollados. Este es un sector que a pesar de la importancia que representa manifiesta problemas que han impedido su mejor desarrollo y crecimiento.

La búsqueda de soluciones alternativas ha generado la necesidad de crear nuevos modelos de producción que fortalezca el desarrollo del sector agrícola y combata factores de influencia negativa como los recursos naturales, la crisis social y ambiental, el dilema de la dependencia alimentaria.

Iris González (2012) quien realizó una investigación con relación a los modelos alternativos de producción y desarrollo agrícola plantea que los modelos de producción y desarrollo agrícola deben comprender un esquema de producción con características bien definidas. De este modo, será más fácil conocer su viabilidad y sustentabilidad pata el desarrollo y crecimiento del sector, y asegurar que sus técnicas productivas armonicen con la optimización de los recursos y el medio ambiente. (González, 2012).

Esta autora también destaca que un modelo de producción agrícola es un proceso productivo de alimentos para satisfacer necesidades humanas, y las demandas de un mercado determinado. Además, un modelo de producción agrícola es aquel que comprende una serie de características bien definidas para su aplicación. Entre ellas diversos factores de gran complejidad para ser viable en cada región o localidad y factores de modelo de producción agrícola como el social, económico, ecológico y cultural que determinan el grado de viabilidad del modelo de producción. El factor social hace posible que todo proceso de desarrollo signifique un crecimiento estable. El factor económico consiste en el flujo constante de inversión pública y privada, la asignación y el manejo eficiente de recursos y un entorno externo justo. El factor ecológico plantea la preservación del medio ambiente y la optimización de los recursos naturales. Y el factor cultural en donde el proceso de modernización debe tener raíces endógenas, buscando el cambio dentro de la continuidad cultural. (Sasch \& Ignacy, 1989) citado en (González, 2012).

Existen diferentes modelos agrícolas los cuales pueden variar en dependencia de la siembra, la fertilización utilizada, el tipo de cosecha, la comercialización utilizada y otros, pero en su concepción básica como modelo todos deben coincidir en su objetivo el cual gira en torno a la producción de bienes con menores costos y máxima calidad para hacer frente a las competencias existentes del mismo sector.

Según estudios revisados se plantea que actualmente existen o se conocen cuatro modelos principales para el sector agrícola. Dentro de ellos se encuen- 
tran el modelo de agricultura convencional el cual se caracteriza por el fortalecimiento del trabajo de la tierra, el modelo convencional empresarial donde se introduce la mecanización para el trabajo en el campo, el modelo de biotecnología a partir del cual se logran cambios en el modo de pensar y se busca el incremento de las ganancias con la reducción de los costos, y el modelo de producción orgánica con varios sistemas de producción agrícola entre los cuales se encuentran el bienestar social, económico, político, cultural y ecológico.

En opinión de la investigadora Iris González (2012) los modelos de agricultura convencional tienen como operación principal la labranza del suelo en su proceso productivo. Es decir, que la labranza del suelo se da a través del arado, el cual se ha convertido en la herramienta más ampliamente utilizada para la realización de esta actividad productiva y como un símbolo de producción agrícola.

El modelo de agricultura convencional empresarial surge como necesidad de transición a las agriculturas óptimas, a través de una creciente mecanización del campo y la selección de nuevas variedades y razas adaptadas a las nuevas condiciones de cultivo. Este modelo propició el alza creciente de la industria agrícola para lograr un comercio cada vez más activo de productos agrícolas primarios y elaborados.

El modelo de biotecnología surge como herramienta para obtener mayores ganancias de las actividades agrícolas tras la reducción de los costos de producción. Esto convirtió a la producción de alimentos en la última mitad de esta década en una cuestión de política comercial que lo abarca todo. (González, 2012)

Estos modelos a su vez presentan fortalezas y debilidades que hacen del modelo una herramienta que aunque resulta beneficioso también afecta y provoca daños en determinadas circunstancias.

La agricultura tradicional a largo plazo provoca daños ambientales deteriorando el suelo y su capa vegetal, causando también erosión, disminución de la fertilidad y pérdida de biodiversidad ambiental. Estas afecciones han provocado en países como Brasil, en Norteamérica, nueva Zelandia y Australia la creación de movimientos a favor de la conservación del suelo. Otra de las características de este tipo de modelo consiste en su lento progreso ya que su realización se desarrolla en pequeños espacios.

El modelo convencional empresarial beneficia a aquellos productores con extensiones grandes de tierra donde pueden ser empleadas maquinarias modernas que fortalezcan la producción. Con este tipo de modelo se incrementan las producciones al incluirse el uso de fertilizantes y productos químicos lo cual favorece la producción pero a su vez ataca el suelo y con ello el medio ambiente.

El modelo de biotecnología posibilitó la obtención de precios más favorables para la adquisición de medios para la producción y también la obtención de grandes beneficios en la venta de productos. Con la aplicación de este 
modelo se mejoró la generación de alimentos potenciando el cultivo libre de productos químicos.

La biotecnología reduce en buena medida el uso de agroquímicos para la obtención de buenas cosechas. Sin embargo, es vista como una amenaza, ya que puede generar fuertes desequilibrios sociales, políticos y económicos de cada país en desarrollo.

No todos los resultados obtenidos con esta práctica han sido beneficiosos ya que ha provocado consecuencias negativas debido a la pérdida de biodiversidad en el medio ambiente provocando rompimiento en el desequilibrio ecológico. "El modelo de producción biotecnológico genera beneficios u oportunidades para el desarrollo del sector agrícola. Sin embargo, estos beneficios son captados por unos pocos empresarios, siendo en buena medida excluyente". (Obbelink \& Henk, 1987)

El modelo de producción orgánica ofrece un sin números de ventajas tanto social, económico, político y ambiental. Posibilita la obtención de mejores precios en los productos producidos por los campesinos. Protege a los trabajadores garantizando un trabajo ambientalmente seguro previniendo la aparición de enfermedades que provocan los productos químicos que son utilizados en la agricultura convencional.

\subsection{Modelos asociativos en el sector agroalimentario. Principales ventajas}

Los autores Vinicio Murillo \& Salas (2008) definen la asociatividad como "un mecanismo de cooperación entre empresas pequeñas y medianas, en donde cada empresa participante, manteniendo su independencia jurídica y autonomía gerencial, decide voluntariamente participar en un esfuerzo conjunto con los otros participantes para la búsqueda de un objetivo común.

Destacan además, cuatro características principales:

- La asociatividad o grupos empresariales asociativos permiten resolver problemas conjuntos manteniendo la autonomía gerencial de las empresas participantes.

- La asociatividad puede adoptar diversas modalidades jurídicas y organizacionales.

- No excluye a ninguna empresa por el tipo de mercado en el cual opera.

- Es exclusiva para pequeñas y medianas empresas. (Vinicio Murillo, Salas, \& Condo, 2008)

La asociatividad posee grandes ventajas dentro de la cuales se pueden mencionar:

- Acceso a financiamiento. Esta ventaja es muy significativa, especialmente cuando las garantías que se requieren no pueden ser cubiertas por cada actor en forma individual, pero en grupo, son cubiertas proporcionalmente por cada uno de los participantes. 
- Compras conjuntas. Debido al tamaño de las operaciones, el volumen que representan sus compras de materia prima o algunos insumos pueden representarles altos costos o simplemente la imposibilidad de adquirirlos. Las compras conjuntas son un mecanismo comúnmente utilizado, que brinda opciones más económicas y viables de adquirir sus insumos.

- Inversiones conjuntas. Al igual que en el caso anterior, la asociatividad representa una opción viable para que un grupo de empresas realicen inversiones, logrando así adquirir diversos activos que individualmente no podrían tener. Esta posibilidad representa una ventaja competitiva muy importante, asociada principalmente con la adquisición de nuevas tecnologías e innovación de los procesos.

- Capacitación conjunta. Por medio de las asociaciones, es factible alcanzar capacitación, de forma que los costos sean cubiertos proporcionalmente. De esta manera se logra la accesibilidad de este tipo de empresas a expertos en diferentes temas de interés conjunto.

- Acceso a mercados. Una de las principales razones de asociación puede ser para mejorar o alcanzar acceso a diferentes mercados. La ventaja de la asociatividad en este tema se relaciona con la accesibilidad a las inversiones necesarias en cuanto a estudios de mercado y promoción en el destino escogido para vender. (Vinicio Murillo, Salas, \& Condo, 2008)

En informe realizado por el autor (Villareal, 2013) al XII Encuentro Nacional de Porcicultura, expone los grandes obstáculos existentes hoy para el desarrollo y la productividad en el sector agroalimentario, proponiendo como solución el desarrollo de clúster de agronegocios, lo cual se puede definir también como la creación de grupos asociativos en el sector agroalimentario. La creación de clúster señala la solución del pequeño productor mediante los grupos asociativos, lo cual potenciaría la productividad y mayor economía. De modo general, estos representan un ejemplo de esquema para el diseño de un modelo asociativo en el sector agroalimentario. En ellos se muestra la asociatividad de productores, los cuales se integran a través de grupos o cooperativas que tienen como incentivos el financiamiento, insumos, la comercialización, tecnología y capacitación y sanidad e inocuidad, todo integrado a la cadena de valor.

\subsection{El rol de la innovación y la gestión del conocimiento para el desarrollo} local sostenible del sector agroalimentario ecuatoriano. Consideraciones en torno a buenas prácticas de gestión y encadenamientos productivos

El hombre necesita del conocimiento para llevar a cabo las transformaciones que implican el desarrollo. Muchas veces el ser humano se encuentra inmerso en una compresión ingenua de aquello que ocurre a su alrededor, de ahí la necesidad de superación y el paso adelante en la gestión del conocimiento lo cual implica un pensamiento transformador y progresivo. 
Como plantea el autor Freire, el conocimiento exige una presencia curiosa del sujeto frente al mundo. Requiere su acción transformadora sobre la realidad. Demanda una búsqueda constante. Implica invención y reinvención. Reclama la reflexión crítica de cada uno sobre el acto mismo de conocer, por el cual se reconoce conociendo y al reconocerse así, percibe el cómo de su conocer, y los condicionamientos a que está sometido su acto. (Freire, 2015)

La transformación de la información puede ser un modo de elaborar la percepción de aquello que se conoce o lo que preliminarmente se observa. Las personas se sienten partes del mundo en que viven o son admiradores de todo aquello que los rodea como un ser independiente capaz de reaccionar ante toda actividad.

El autor mencionado anteriormente también manifiesta la relación entre el pensamiento y la percepción de la información lo cual implica el conocimiento sobre determinado tema y como se comprende. Expresa que

El pensamiento mágico no es ilógico, ni es prelógico. Tiene su estructura lógica interna, y reacciona, hasta donde puede, al ser sustituido mecanicistamente por otro. Este modo de pensar, como cualquier otro, está indiscutiblemente ligado tanto a un lenguaje y a una estructura, como a una forma de actuar. (Freire, 2015)

El sector agrario o campesino lo conforman grupos de personas que demandan conocimientos tecnicistas o procedimientos específicos de industrialización y cambiar sus métodos o modo de pensar y de comprender la realizad a su alrededor debe ser con tacto y manejo accequible para no resultar invasores de su espacio.

Al respecto (Freire, 2015) señala que sobreponer a ellos otra forma de pensar, que implica nuestro lenguaje, nuestra estructura y nuestra manera de actuar, despierta una reacción natural. Una reacción de defensa ante el invasor que amenaza romper si equilibrio interno.

La acción del hombre ante el mundo es la posición de admirador capaz de reaccionar y reflexionar sobre él. Analizando el actuar de los campesinos se puede comprender su posición frente a los cambios y transformaciones de su trabajo.

Cuanto más observamos las formas de comportarse y de pensar de nuestros campesinos, más parece que podemos concluir que, en ciertas áreas en mayor o menor grado, se encuentran, de tal forma próximos al mundo natural, que se sienten más como parte de él, que como sus transformadores. (Freire, 2015)

Independientemente de las características y pensamientos de sectores de población o grupos de trabajo resulta un avance integrador el desarrollo local a través de los avances de la innovación y el conocimiento.

De esta forma, con el conocimiento como factor movilizador de los capitales se configuran nuevos paradigmas de intervención para la promoción del 
desarrollo regional, donde los elementos claves son: la interconexión y la interactividad, la acción colectiva, la escala geográfica reducida, la escala virtual amplia, el constructivismo, la intersubjetividad, el conocimiento y las innovaciones. A su vez, estos elementos necesitan como factor promotor la confianza y creación de varias formas de capital colectivo. (Sánchez Zambrano, Escobar Jaramillo, Sánchez Mejía, \& Concha Arango, 2013)

A su vez, la innovación como sistema busca la interacción entre instituciones para generar conocimientos respaldado por la asignación de recursos que facilitan la divulgación de los soportes innovadores como base del desarrollo económico.

Así, en el sistema se conforma una red de organizaciones o actores claves, que constituyen el origen para que las sociedades creen, almacenen y transfieran conocimiento, habilidades y artefactos, que contribuyen con la innovación. Desde esta perspectiva, el desempeño en innovación de una economía depende no sólo de cómo se comportan las organizaciones individuales aisladamente, sino también de cómo interactúan entre sí, y de su interacción con instituciones sociales como los valores, normas y marcos legales. Organización para la Cooperación y Desarrollo Económico (2007).

Los autores de la ponencia coinciden al expresar que las potencialidades de un territorio y el desarrollo de su economía basado en sistemas de innovación necesitan del fortalecimiento de factores importantes como el conocimiento, lo cual actualmente se encuentra poco explotado sin aprovechar la oportunidades que se obtienen a través de la gestión del mismo. Se debe fortalecer la creación de sistemas de innovación que fortalezcan los diferentes sectores de la economía y con ello el desarrollo local. De este modo se promueven cambios estructurales, los cuales implican los diferentes sectores de la sociedad como sectores productivos e instituciones en general.

La gestión del conocimiento y la innovación resultan estrategias básicas para el desarrollo local, ya que el mismo implica integración entre todas las dimensiones de la sociedad buscando el fortalecimiento de las localidades a través de su economía y potencialidades naturales, respetando las fortalezas de las regiones y su cultura local.

Hablar de desarrollo local es aludir a "ese conjunto de procesos económicos, sociales, culturales, políticos y territoriales a través de los cuales una comunidad, a partir de sus propias potencialidades y de las oportunidades que le brinda el entorno, accede al bienestar, sin exclusiones ni discriminaciones, y garantiza las condiciones para que futuras generaciones también puedan hacerlo. (Carvajal Burbano, 2011)

Si la importancia del desarrollo se apoya como base en el conocimiento se promueve una integración de comunidades donde se aprende de las experiencias de cada lugar, lo cual les permite compararse y proyectarse en el desarrollo y el camino a tomar para encontrar un mejor futuro. 
El desarrollo local debe emprenderse teniendo en cuenta la sostenibilidad y bajo el concepto de desarrollo local sostenible. Concepto que ha sido manejado por diferentes autores los cuales han manifestado su criterio al respecto

Un proceso de crecimiento y cambio estructural de la economía de una ciudad, comarca o región, en el que se pueden identificar, al menos, tres dimensiones: una económica, caracterizada por un sistema de producción que permite a las empresas locales usar, eficientemente, los factores productivos, generar economías de escalas y aumentar la productividad a niveles que permitan mejorar la competitividad en los mercados; otra sociocultural, en que el sistema de relaciones económicas y sociales, las instituciones locales y los valores, sirven de base al proceso de desarrollo, y otra político-administrativa en que las iniciativas locales crean un entorno local favorable a la producción e impulsan el desarrollo sostenible. (Morales Pérez, 2006)

Los autores consideran además, que puede verse como "un proceso de objetivos múltiples, que son: eficiencia en la asignación de recursos para la competencia territorial; equidad en la distribución de la renta y equilibrio del entorno medioambiental para la conservación del sistema productivo territorial".

Estos conceptos enmarcan la importancia del nuevo pensar de las empresas, las cuales además de preocuparse por su producción y economía deben trazar estrategias que impliquen el desarrollo basado en el concepto de la sustentabilidad, elaborando productos aceptados ambientalmente, reduciendo el consumo de materiales y los desechos innecesarios, aprovechamiento al máximo de los recursos y preservación del entorno donde se encentra.

En el Ecuador se potencia el sistema agroalimentario basado en la sostenibilidad combinando la industrialización con un proceso productivo que apoye las técnicas de producción sustentables. En los últimos años el desarrollo capitalista ha potenciado la producción, buscando incrementos sin tener en cuenta los recursos naturales y la preservación de estos. Unido a un sistema de cultivo donde los agroquímicos y pesticidas son elemento fundamental del proceso de cultivo.

El 30 de mayo de 2014 se celebró en Quito la mesa sobre agricultura sostenible durante la Cumbre del Buen Conocer donde se propusieron medidas concretas para facilitar la adopción de un régimen de agricultura sustentable como medio para que el sector agrícola cumpla un papel preponderante en la transición hacia la economía social del conocimiento común y abierto. En dicho régimen, la aplicación de los principios agroecológicos asegura la sostenibilidad, al integrar en la producción los procesos ecológicos y los controles biológicos, así como hacer uso de los recursos disponibles a bajo coste y a escala local. Por otro lado, este régimen intensifica el rol del conocimiento, en la medida en que exige relanzar procesos de innovación destinados a sustituir los insumos privativos, suministrados oligopólicamente y con merma de la soberanía alimentaria, por la articulación de las técnicas y recursos tradicionales con los últimos 
avances de la investigación científica abierta y colaborativa, así como con un cambio profundo en la cultura sobre la producción y el consumo agroalimentarios. Por último, la eficacia de esta transición requiere el fortalecimiento del sector de pequeños productores-consumidores y de su institucionalidad propia. En este sentido, la agricultura sustentable se autoorganiza mediante la gestión cotidiana de las comunidades, a través de redes institucionales crecientemente tupidas y compuestas por mercados locales, bancos y redes de compartición de semillas y otros recursos, escuelas técnicas, empresas de la economía popular y solidaria, instituciones de financiamiento y redes de distribución participadas por productores y consumidores. (Dafermos \& Vivero Pol, 2015)

Como se puede apreciar en el Ecuador se trabaja en función del desarrollo sostenible, vinculando la gestión del conocimiento y la innovación encaminando sistemas productivos en el sector agroalimentario que potencien el desarrollo sustentable. En consideración a esto se estudian estrategias y proyección de modelos en el sector agroalimentario que implique el desarrollo a través del manejo de buenas prácticas de gestión en la producción.

Actualmente, se han aplicado modelos alternativos para un sistema agroalimentario sostenible lo cual ha proporcionado beneficios ambientales y socioeconómicos para los productores y la población en general. Estos modelos han seguido las premisas siguientes:

- Aplicación de los principios ecológicos y agroecológicos. Por ejemplo, integra los procesos ecológicos y los controles biológicos como equilibrar el nitrógeno, completar el ciclo de nutrientes o el de regeneración de suelos. A la vez, hace un uso de los recursos disponibles a bajo coste y a escala local, como por ejemplo el compost reciclado de jardines y desperdicios de los hogares.

- Intensificación del conocimiento, ya que la agricultura ecológica requiere el desarrollo y la divulgación de prácticas y conocimientos imprescindibles para sustituir los insumos de la agro-industria por las técnicas más tradicionales articuladas en procesos de innovación social.

- Gestión con base en la comunidad. Uno de los principios de esta agricultura es la gestión de los recursos fundamentales para su desarrollo, en relación con las plagas, los recursos hídricos, forestales o la misma gestión financiera, a través de una participación activa de la comunidad en la gestión cotidiana. (Dafermos \& Vivero Pol, 2015).

\section{Conclusiones}

La búsqueda de soluciones alternativas ha generado la necesidad de crear nuevos modelos de producción que fortalezcan el desarrollo del sector agrícola y combata factores de influencia negativa como los recursos naturales, la crisis social y ambiental, el dilema de la dependencia alimentaria. 
Actualmente, existen el modelo de agricultura convencional el cual se caracteriza por el fortalecimiento del trabajo de la tierra, el modelo convencional empresarial donde se introduce la mecanización para el trabajo en el campo, el modelo de biotecnología a partir del cual se logran cambios en el modo de pensar y se busca el incremento de las ganancias con la reducción de los costos, y el modelo de producción orgánica con varios sistemas de producción agrícola entre los cuales se encuentran el bienestar social, económico, político, cultural y ecológico.

La asociatividad como "un mecanismo de cooperación entre empresas pequeñas y medianas, en donde cada empresa participante, manteniendo su independencia jurídica y autonomía gerencial, decide voluntariamente participar en un esfuerzo conjunto con los otros participantes para la búsqueda de un objetivo común.

Existen grandes obstáculos existentes hoy para el desarrollo y la productividad en el sector agroalimentario, proponiendo como solución el desarrollo de clúster de agronegocios, lo cual se puede definir también como la creación de grupos asociativos en el sector agroalimentario. La creación de clúster señala la solución del pequeño productor mediante los grupos asociativos, lo cual potenciaría la productividad y mayor economía.

La gestión del conocimiento y la innovación resultan estrategias básicas para el desarrollo local, ya que el mismo implica integración entre todas las dimensiones de la sociedad buscando el fortalecimiento de las localidades a través de su economía y potencialidades naturales, respetando las fortalezas de las regiones y su cultura local.

Es necesario, transitar hacia un modelo agrícola sostenible que revalorice el valor del campesinado ecuatoriano, logre la inclusión y la participación ciudadana con equidad y justicia social, respete las prácticas agrícolas ancestrales, incremente los puestos de trabajo, incorpore las mejores prácticas de consumo sustentable y sostenible y se centre en la gestión del conocimiento, la innovación y el desarrollo local endógeno.

BibLIOGRAFÍA

CARVAJAL BURBANO, A. (2011): Desarrollo Local: Manual Básico para Agentes de. Málaga: eudmet.net.

DAFERMOS, G., \& VIVERO POL, J. (2015): Agroalimentación: naturaleza y saberes autónomos frente al capitalismo biotech (v.2.0). Documento de política pública . Retrieved from http://book.floksociety.org/ec/2-1-sistemaagroalimentario-abierto-y-sustentable-en-ecuador/

FREIRE, P. (2015): ¿Extensión o comunicació? La concientización en el medio rural. Siglo XXI Editores.

GONZÁLEZ, I. (2012): Modelos alternativos de producción y desarrollo agrícola. Retrieved from http://catarina.udlap.mx/u_dl_a/tales/documentos/lri/ gonzalez_m_pi/capitulo2.pdf 
MORALES PÉREZ, M. (2006): El desarrollo local sostenible. Economía y desarrollo No 2. Vol. 140, 62.

OBBELINK, \& Henk. (1987): Más allá de la Revolución Verde. Las nuevas tecnologías Genéticas de la Agricultura ¿Desafíos o Desastre? Barcelona: Lerna.

ORGANIZACIÓN PARA LA COOPERACIÓN Y DESARROLLO ECONÓMICO. (2007). Estudis de la OCDE sobre políticas de innovación. Chile: Ministerios de Economía Fomento y Reconstrucción de Chile.

OSTERWALDER, A. \&. (2010): Business Model Generation. New Yersey: John Wiley \& Sons.

RUIZ MASSIEU, D. (2011): ¿Modelo de Negocio o Business Plan? Dirección Estratégica 37, 1-2.

SÁNCHEZ ZAMBRANO, K., ESCOBAR JARAMILLO, L. A., SÁNCHEZ MEJÍA, M., \& CONCHA ARANGO, G. (2013): Desarrollo Local Basado en Conocimiento e Innovación:Caso Agrópolis del Norte. Journal of Technology Management \& Innovation, 106.

SASCH, \& IGNACY. (1989): Desarrollo sustentable. Nioindustrialización. Descentraliazación y nuevas configuraciones Rural-Urbanas. Los Casos india y Brasil. Madrid: Pensamiento Iberoamericano.

VILLAREAL, R. (2013). El modelo de agroclústers. El Camino a la Productividad. Ixtapa.

VINICIO MURILlO, J., Salas, C., \& Condo, A. (2008). Asociatividad y Competitividad: Factores que influyen en la competitividad de un grupo asociativo. Alajuela: INCAE. 